\title{
REVISIÓN
}

\section{Citocinas en la patogenia de la enfermedad inflamatoria intestinal}

\author{
Alberto José León ${ }^{\mathrm{a}}$, José Antonio Garrote ${ }^{\mathrm{a}, \mathrm{b}}$ y Eduardo Arranz ${ }^{\mathrm{a}}$ \\ aÁreas de Pediatría e Inmunología. Instituto de Biología y Genética Molecular (IBGM). \\ Universidad de Valladolid. Valladolid. \\ bUnidad de Investigación. Hospital Clínico Universitario. Valladolid. España.
}

\begin{abstract}
La enfermedad inflamatoria intestinal (EII) se debe a una respuesta exagerada frente a la flora bacteriana en la mucosa intestinal, en la que intervienen factores ambientales y genéticos. Los linfocitos T actúan tanto en el origen como en el mantenimiento de la Ell, y su patrón de citocinas es diferente en la enfermedad de Crohn, con predominio de citocinas Th1, y en la CU, donde el perfil es Th2. Una vez iniciado el proceso inflamatorio, el balance entre citocinas proinflamatorias y reguladoras determina el grado de afectación y la forma de presentación de la enfermedad. El conocimiento en los mecanismos inmunológicos que intervienen en la EII, ha abierto nuevas líneas de trabajo con fines terapéuticos como son: la neutralización de citocinas proinflamatorias mediante anticuerpos y la administración de citocinas antiinflamatorias, que se encuentran en diferentes fases de investigación.
\end{abstract}

Palabras clave: Citocinas. Linfocitos T. Enfermedad inflamatoria intestinal.

Cytokines in the pathogenesis of inflammatory bowel diseases

Inflammatory bowel disease (IBD) is produced by an exaggerated response to bacterial flora within the intestinal mucous, in which both environmental and genetic factors are involved. T lymphocytes are involved during the genesis and maintenance of IBD, and their cytokine profile in Crohn's disease (mostly Th1 cytokines) is different from that in ulcerative colitis (mainly Th2 cytokines). After the inflammatory response has been established, the balance between proinflammatory and regulatory cytokines determines the degree of mucosal damage and the form of presentation. A deeper knowledge of the immunological mechanisms involved in IBD has opened new research lines aimed to the development of new therapies such as the neutralization of proinflammatory cytokines with antibodies and the administration of antiinflammatory cytokines, which are currently at different stages of research.

Key words: Cytokines. T lymphocytes. Inflammatory bowel diseases.

La enfermedad inflamatoria intestinal (EII) es un trastorno caracterizado por una inflamación crónica del tracto intestinal que evoluciona de modo recurrente en forma de brotes y que se manifiesta como una o dos entidades clínicas normalmente distintas, colitis ulcerosa (CU) y enfermedad de Crohn (EC), aunque a veces se expresan de forma solapada. La primera afecta al colon y es una enfermedad ulcerati-

El trabajo en nuestro laboratorio está financiado con ayudas del Instituto de Salud Carlos III, Ministerio de Sanidad (PI020895, 02/3068), Junta de

Castilla y León (VA057/04), becas de Formación de Personal Investigador de la Junta de Castilla y León (O.C.-14/11/03-O.R.-26/01/0), y Sweden Diagnostics afiliada a Pharmacia Diagnostics.

Correspondencia: Dr. E. Arranz.

Áreas de Pediatría e Inmunología. Instituto de Biología y Genética Molecular (IBGM). Universidad de Valladolid.

Ramón y Cajal, 7. 47005 Valladolid. España.

Correo electrónico: earranz@med.uva.es

Recibido el 10-11-2005; aceptado para su publicación el 16-12-2005. va superficial, mientras que la segunda es una enfermedad transmural de carácter granulomatoso que tiene predilección por el íleon terminal y el colon, aunque puede afectar a cualquier parte del tubo digestivo ${ }^{1}$. Ambas formas pueden asociarse con manifestaciones extraintestinales prominentes, como fiebre, pérdida de peso, retraso en el crecimiento, artralgias o artritis, lesiones mucocutáneas y una mayor incidencia de cáncer gastrointestinal, que se da especialmente en la $\mathrm{CU}^{2}$. Además, estas enfermedades se presentan relativamente pronto en individuos jóvenes y persisten durante largos períodos, afectando de forma importante a la calidad de vida y aumentando la morbimortalidad. La EC y la CU podrían considerarse como síndromes heterogéneos que expresan un fenotipo clínico-patológico similar, y comparten determinados factores de riesgo genéticos y ambientales, pero difieren en otros ${ }^{3}$.

\section{Causas de la enfermedad inflamatoria intestinal}

Los datos experimentales y observacionales indican que la inflamación intestinal surge de una reacción inmunitaria anormal a la flora bacteriana en el intestino de personas genéticamente susceptibles (fig. 1 ) $^{4}$. Sin embargo, la utilidad probada de los antibióticos en determinados grupos de pacientes con $\mathrm{EC}^{5}$, frente a su limitada eficacia en la CU, indica que el papel que desempeña la flora en los dos tipos de inflamaciones puede ser diferente ${ }^{6}$. Aunque no pueden excluirse patógenos específicos, el origen de la enfermedad no puede explicarse únicamente por la acción de ciertos microorganismos en el sentido tradicional de una infección entéri$\mathrm{ca}^{3}$. El estudio en las interacciones huésped-flora se ve frenado por los vacíos en el conocimiento de la flora normal, ya que al menos la mitad de las especies de bacterias de la flora no se puede cultivar y los estudios que muestran posibles cambios cualitativos son inconsistentes o contradictorios ${ }^{7}$.

Diversos elementos en el medio ambiente podrían afectar al desarrollo del sistema inmunitario de la mucosa gastrointestinal y a la composición de la microflora, como por ejemplo, una mayor higiene, el consumo de comida no estéril fermentada, la vacunación y la edad de la primera exposición a patógenos intestinales ${ }^{3}$. El descenso en la frecuencia de infecciones por helmintos observada en el mundo más desarrollado forma parte de esta hipótesis de la higiene, y se ha asociado mecanísticamente con el aumento en la prevalencia de la $\mathrm{EC}^{8}$. Otro factor relacionado con el modo de vida y que influye decisivamente en la patogenia de la Ell es el estrés, que hasta el $40 \%$ de los pacientes con CU lo sufre ${ }^{9}$. Además de la influencia del medio ambiente, diversos factores genéticos aumentan el riesgo de desarrollar Ell, y éstos resultan más importantes en la EC que en la CU, como demuestra la mayor concordancia en gemelos homocigotos encontrada en la primera (44-50\%) con respecto a la segunda $(6-14 \%)^{10,11}$. Ambos trastornos comparten algunos genes de susceptibilidad, mientras que otros son específi- 


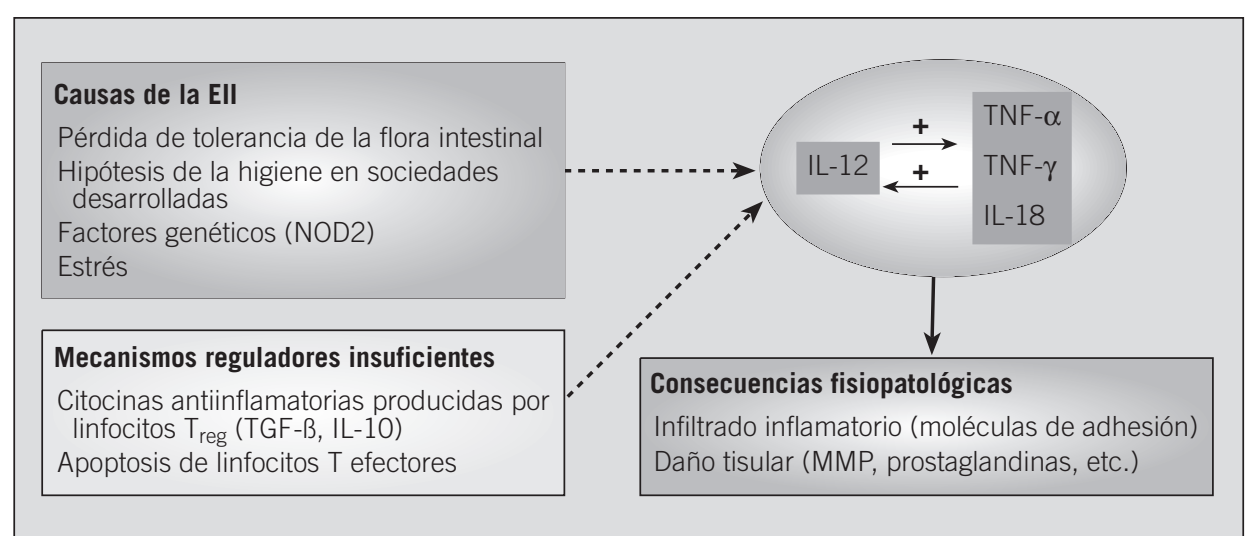

Fig. 1. Los factores ambientales y genéticos, junto con un déficit en los mecanismos reguladores, desencadenan la Ell. Varias citocinas proinflamatorias actúan de forma sinérgica induciendo el infiltrado inflamatorio y el daño tisular. Ell: enfermedad inflamatoria intestinal; NOD: gen asociado con mayor susceptibilidad de presentar la enfermedad de Crohn; IL: interleucina; IFN$\gamma$ : interferón gamma: TNF- $\alpha$ : factor de necrosis tumoral alfa; Treg: linfocitos $T$ reguladores; TGF- $\beta$ : factor transformador del crecimiento beta; MMP: metaloproteinasas. cos de la EC o de la CU y determinan factores como la gravedad $^{12}$, forma de presentación ${ }^{13}$ y el grado de respuesta al tratamiento con glucocorticoides ${ }^{14}$. De todos los genes estudiados hasta el momento, el NOD2 es el que presenta una mayor asociación con la EC, aunque también se han realizado estudios en los genes del HLA (anticuerpos leucocitarios humanos) y del antagonista del receptor de la interleucina 1 (IL-1-Ra), entre otros ${ }^{3}$.

\section{Inicio de la enfermedad: activación de las células T de la mucosa}

En el hombre, la respuesta inmunitaria normal del intestino está dirigida hacia la vía Th $1^{15}$, aunque probablemente esto puede variar dependiendo de la implicación de otros factores genéticos y ambientales, incluida la composición de la flora $^{3}$. En condiciones normales, la actividad inflamatoria está regulada por el balance de citocinas producidas por los linfocitos T de la lámina propia mucosa con actividad proinflamatoria, como el interferón gamma (IFN- $\gamma$ ), el factor de necrosis tumoral alfa (tumor necrosis factor alpha, TNF- $\alpha$ ) y la interleucina (IL) 4, así como sus contrapartidas de moléculas antiinflamatorias, factor transformador del crecimiento beta (transforming growth factor beta, TGF- $\beta$ ) e IL-10, de tal forma que se orquesta una respuesta eficaz frente a las agresiones en el ámbito local, al tiempo que se mantiene el nivel de actividad inflamatoria dentro de los valores fisiológi$\cos ^{16}$.

Los linfocitos T de la lámina propia, que son en último término la causa de la regulación de la inflamación intestinal, se encuentran en la Ell en un elevado grado de activación y presentan una mayor resistencia a la apoptosis, lo que conduce a una acumulación local de células $T$ y a la perpetua- ción de la respuesta inflamatoria ${ }^{17}$. Esta resistencia se ha atribuido a la IL-6, ya que estimula la cascada de expresión de genes de acción antiapoptótica en células T del intestino ${ }^{18}$. En cuanto a la población de células T CD8+ aislada de la lámina propia, que es comúnmente considerada como citotóxica, no presenta un alto grado de activación en la Ell, como demuestra el hecho de que el nivel de expresión de perforina es semejante al encontrado en los controles sanos $^{19}$. Por el contrario, en la Ell se encuentran presentes poblaciones CD4+ con acción citotóxica, situación que sólo se encuentra en situaciones patológicas y no en condiciones normales. El efecto citotóxico de estas poblaciones CD4+ tiene importancia por las interacciones que éstas mantienen con las células que presentan el antígeno, y que podría ocasionar un daño directo en determinadas poblaciones, como los enterocitos ${ }^{19}$.

Hay poblaciones de linfocitos $T$ reguladores encargados de suprimir la respuesta de otras poblaciones de linfocitos $T$ efectores mediante la producción de citocinas de acción supresora ${ }^{20}$. Se ha visto que las poblaciones de células CD4+CD25 (Bright) de la lámina propia ejercen una función supresora en la proliferación y la producción de citocinas de otros linfocitos T. A pesar del aumento de estas poblaciones supresoras en la lámina propia de pacientes con EC, el efecto de estas células podría no ser suficiente para controlar la inflamación ${ }^{21}$

Además de un mayor nivel de activación, los linfocitos T presentes en la lámina propia en pacientes con Ell producen diferentes perfiles de citocinas, lo que conduce en último término a la activación de mecanismos fisiopatológicos diferentes. Según Fuss et $\mathrm{al}^{22}$, los linfocitos $\mathrm{T}$ de la lámina propia activados in vitro ofrecen una respuesta distinta según procedan de pacientes con EC o CU19,22-25 (tabla 1): en

\section{TABLA 1}

\section{Resumen de los estudios más relevantes sobre el perfil de citocinas producido por linfocitos T de la lámina propia procedentes de biopsias de intestino, tanto en pacientes con enfermedad de Crohn como de colitis ulcerosa*}

\begin{tabular}{|c|c|c|c|}
\hline Referencia & Estimulación & Población celular estudiada & Expresión de citocinas (respecto controles) \\
\hline Agnholt y Kaltoft²3 & IL-12, superantígeno & Células T de LP cultivo con IL-2 + IL-4 & Crohn: $\uparrow$ IFN- $\gamma$, 个TNF- $\alpha, \uparrow I L-10$ \\
\hline Fuss et al ${ }^{22}$ & Vía CD2/CD28 & Células T de LP & $\begin{array}{l}\text { Crohn: } \uparrow \text { IFN- } \gamma, \downarrow \text { IL-2, } \downarrow \text { IL-4, } \downarrow \text { IL-5 } \\
\text { CU: } \uparrow \text { IL-5, = IFN- } \gamma, \downarrow \text { IL-4 }\end{array}$ \\
\hline Parronchi et $\mathrm{al}^{24}$ & $\mathrm{PMA}+$ anti-CD3 & Clones derivados de células T de LP & $\begin{array}{l}\text { Crohn: IFN- } \gamma \text { muy frecuente, poco IL-4 } \\
\text { CU y control: en bastantes clones } \\
\text { simultáneamente IFN- } \gamma \text { e IL-4 }\end{array}$ \\
\hline Van Damme et $a^{25}$ & PHA-ionomicina & Células T de LP & $\begin{array}{r}\text { Crohn: } \downarrow \text { IFN- } \gamma, \downarrow \text { IL-2 } \\
\text { CU: } \downarrow \text { IFN- } \gamma, \downarrow \text { IL-2 }\end{array}$ \\
\hline Mariani et al ${ }^{19}$ & PMA + anti-CD3 & Células T de LP & Crohn: $\uparrow$ IFN- $\gamma,=$ IL-2, $\downarrow$ IL-4, $\uparrow$ TNF- $\alpha$ n.d. IL-5 \\
\hline
\end{tabular}

CU: colitis ulcerosa; IL: interleucina; LP: lámina propia; IFN- $\gamma$ : interferón gamma; TNF- $\alpha$ : factor de necrosis tumoral alfa; PMA: phorbol myristate acetate; PHA: fitohemaglutinina. *Algunas diferencias en los resultados pueden deberse a las distintas aproximaciones experimentales utilizadas. 
los primeros se observa un gran aumento en la producción de IFN- $\gamma$ debida a una gran expansión en el número de células que lo sintetizan, al tiempo que se produce una pequeña disminución en los valores de IL-4 e IL-5. También se produciría una importante disminución de IL-2, por lo que se trataría de una respuesta Th1 no pura debido a la ausencia de esta citocina, aunque este resultado contradice algunos estudios anteriores. En el caso de la CU, no se modifican los valores de IFN- $\gamma$ en comparación a los encontrados en los controles sanos, se produce una pequeña disminución de la IL-4 y un gran aumento de la IL-5, que se incrementa incluso en condiciones basales, sin estimulación. Otros estudios han llegado a conclusiones similares utilizando diferentes aproximaciones ${ }^{19,22-25}$ y, aunque el papel de la IL-2 en la EC resulta contradictorio, puede afirmarse que en esta enfermedad los linfocitos $T$ producen un patrón de citocinas de tipo Th1 (IFN- $\gamma$ y TNF- $\alpha$ ), mientras que en la CU la respuesta es de tipo Th2 (IL-5) ${ }^{4}$.

\section{Inflamación grave: reclutamiento de macrófagos y producción de mediadores inflamatorios}

Cuando se estudian las biopsias completas, tanto de EC como de CU, se encuentra un aumento en las concentraciones de citocinas proinflamatorias IL-1 $\beta$, IL-2, IL-6, IL-8, IL-15, IL-18, IFN- $\gamma$, TNF- $\alpha$, y los miembros de la familia del IL-12 (IL-12, IL-23, IL-27), entre otros ${ }^{26}$. A pesar de que hay diferencias en el perfil de citocinas de los linfocitos T activados en la EC y la CU, causantes del inicio y del mantenimiento de la inflamación, una vez que se produce el reclutamiento de macrófagos y de linfocitos de sangre periférica muchos de los mediadores inflamatorios son comunes a ambos trastornos ${ }^{26}$. Además del incremento en los valores de citocinas inflamatorias, también aumentan sus oponentes antiinflamatorios, tanto en la Ell como en otros procesos inflamatorios de la mucosa intestinal, y el equilibrio entre ambos grupos es lo que finalmente determina el curso de la enfermedad ${ }^{16}$.

Las células recién recogidas que se encuentran en la mucosa se encuentran en un elevado grado de activación, y son los principales efectores de la respuesta inmunitaria celular, así como los mayores productores de citocinas ${ }^{27}$. Del número total de células productoras de citocinas en el tejido de Ell, la mayoría son macrófagos y un menor número de ellos son linfocitos, aunque hay otras poblaciones celulares que también producen citocinas, como las células epiteliales ${ }^{27}$. A diferencia de otras inflamaciones crónicas del intestino, donde la actividad inflamatoria se encuentra incrementada únicamente dentro de la zona morfológicamente alterada, varias observaciones demuestran que en la EC la actividad inflamatoria se extiende a áreas mucho más amplias ${ }^{28}$, como es la mayor densidad de células plasmáticas en la lámina propia con aumento del volumen de ésta ${ }^{29}$, menor actividad de la disacaridasa ${ }^{30}$, aumento de secreción de proteínas del complemento en el ámbito local ${ }^{31}$, mayor actividad de fosfolipasa $A_{2}{ }^{32}$ y alteraciones leves detectadas por microscopia electrónica en áreas que presentan histología aparentemente normal ${ }^{33}$. Asimismo, otros estudios demuestran la presencia de mayores valores de citocinas inflamatorias en esas zonas que, además, correlacionan con una mayor incidencia en las recaídas tempranas ${ }^{34}$.

\section{Principales citocinas del infiltrado inflamatorio}

Durante el proceso inflamatorio que tiene lugar en la mucosa intestinal, se liberan distintos mediadores inflamatorios que aunque pueden ser producidos por tipos de células dis-

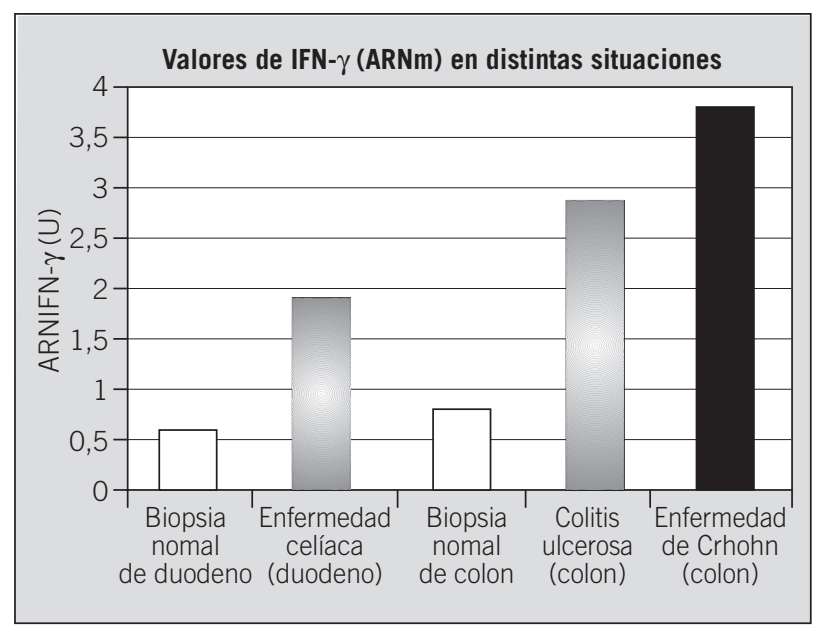

Fig. 2. Los valores de algunas citocinas inflamatorias, como IFN- $\gamma$, aumentan en diversos procesos entre los que se encuentran la enfermedad celíaca, colitis ulcerosa y enfermedad de Crohn, y este incremento es proporcional a la gravedad del daño tisular. El aumento en los valores del IFN- $\gamma$ se produce tanto en trastornos del intestino delgado como del grueso (observaciones propias, resultados en preparación). IFN- $\gamma$ : interferón gamma, ARNm: ARN mensajero, U: unidades arbitrarias.

tintos y actuar en ocasiones en poblaciones celulares diferentes, se secretan dentro de la misma cascada inflamatoria y en muchas ocasiones actúan de forma sinérgica.

El IFN- $\gamma$ es el principal inductor de la actividad bactericida de los macrófagos y estimula la expresión de las moléculas del complejo mayor de histocompatibilidad (major histocompatibility complex, $\mathrm{MHC}$ ) de clases I y II, así como de moléculas coestimuladoras, en las células presentadoras de antígeno, como macrófagos y células dendríticas. Mientras que en la inmunidad innata las células natural killer (NK) producen el IFN- $\gamma$ en respuesta al reconocimiento de ciertos componentes de microorganismos, en la adaptación las células $\mathrm{T}$ producen IFN- $\boldsymbol{\gamma}$ en respuesta al reconocimiento del antígeno, y en ambos casos la IL-12 potencia su producción ${ }^{35}$. Asimismo, una vez que el proceso inflamatorio está en marcha, los macrófagos y los linfocitos T se estimulan mutuamente mediante la producción de IFN- $\gamma$ y de IL12 , respectivamente ${ }^{35}$. Otro efecto del IFN- $\gamma$ es inducir la diferenciación de las células T CD4+ vírgenes hacia el fenotipo Th1 y, además por el contrario inhiben la proliferación de la subpoblación Th2 ${ }^{35,36}$. Se ha observado que el IFN- $\gamma$ es uno de los mediadores más importantes de la inflamación en el intestino, tanto en la patología humana como en los modelos experimentales en animales, y su expresión se ha relacionado directamente con el daño tisular en varios procesos inflamatorios del intestino (fig. 2) ) $^{37,38}$. Se ha observado que los valores de IFN- $\gamma$ se encuentran elevados en la EII, respecto a los controles sanos, tanto la expresión del ácido ribonucleico mensajero (ARNm) ${ }^{26}$ como de proteínas, y se ha detectado mediante inmunohistoquímica un incremento en el número de células productoras de IFN- $\gamma$ de la lámina propia en ambos trastornos, en su mayoría macrófagos y en menor proporción linfocitos, mientras que el número total es superior en la EC respecto a la $\mathrm{CU}^{27}$. Los efectos biológicos del IFN- $\gamma$ dependen de la actividad de una cascada de factores activadores de la transcripción, entre los que se encuentra el signal transducer and activator of transcription (STAT) 1, que regulan la expresión génica al unirse a las regiones promotoras en el núcleo ${ }^{39}$. Se ha visto que los valores de p-STAT1 (la forma fosforilada que presenta actividad) están elevados tanto en la EC como en la CU, y el 
LEÓN AJ ET AL. CITOCINAS EN LA PATOGENIA DE LA ENFERMEDAD INFLAMATORIA INTESTINAL

tratamiento con glucocorticoides tiene un efecto directo en la disminución de p-STAT1, prueba indirecta del importante papel que desempeñan las concentraciones de IFN- $\boldsymbol{\gamma}$ tanto en el desarrollo del trastorno como en la remisión de la enfermedad 40

Principalmente los macrófagos activados producen el TNF$\alpha$, que interviene en múltiples actividades como la acumulación de neutrófilos, formación de granulomas, inducción de la expresión de moléculas de adhesión en el endotelio y otros muchos efectos ${ }^{35}$. Cuando el TNF- $\alpha$ actúa en la mucosa intestinal, incrementa de forma notable su permeabili$\mathrm{dad}^{41} \mathrm{y}$, al tener efectos citostáticos en las células epiteliales, puede inducir la apoptosis de los enterocitos cuando actúa sinérgicamente con el IFN- $\gamma^{42,43}$. El ARNm del TNF- $\alpha$ aumenta en las biopsias tanto de la EC como de la CU en actividad $^{44}$, aunque este incremento sólo se correlaciona con el grado de lesión en el caso de la $\mathrm{CU}^{45}$ y no en la EC ${ }^{28}$. También se ha visto que entre los pacientes con EC, los que muestran los valores de TNF- $\alpha$ más elevados en las zonas sin afectación histológica tienen mayores probabilidades de experimentar recaídas tempranas de la enfermedad, especialmente cuando este aumento es más pronunciado ${ }^{34}$. Además, se ha visto que los valores del ARNm de TNF- $\alpha$ en pacientes con EC en remisión son superiores a los de los controles sanos, mientras que en los de CU en remisión son similares a los encontrados en individuos sanos ${ }^{45}$. A pesar de que la producción de TNF- $\alpha$ no se correlaciona con el grado de afectación histológica en la EC, sí que lo hace con el nivel de IL-1 $\beta$, que es otra citocina implicada en la inflamación intestinal28. Los macrófagos son la principal fuente de TNF- $\alpha^{46}$, y están localizados en la lámina propia, especialmente en la submucosa perivascular asociada al reclutamiento de nuevas células inflamatorias 28 .

La IL-1 $\beta$ es una citocina con acción inflamatoria, tanto de forma local como sistémica, y comparte muchos efectos con el TNF- $\alpha^{35}$. La producen principalmente los granulocitos y los macrófagos de la lámina propia que se han recogido recientemente ${ }^{46}$, y su expresión es especialmente intensa en las ulceraciones ${ }^{47}$. Se ha demostrado la presencia de la forma activa de IL-1 $\beta$ en la Ell, así como de la enzima conversora de la IL-1 $\beta$ (interleukin $1 \beta$ converting enzyme, ICE), que es necesaria para activar tanto la IL-1 $\beta$ como la $\mathrm{IL}-18^{48}$. Cuando se realiza el cultivo in vitro de biopsias $^{28} 0$ bien de las células mononucleares aisladas de la lámina propia $^{49}$, se observa una correlación entre el grado de afectación de la mucosa y la cantidad de IL-1 $\beta$ detectada en el sobrenadante del cultivo, tanto en la EC como en la CU, Io que indica que las células mononucleares se encuentran en un elevado grado de activación en ambas situaciones. Asimismo, en las biopsias de la EC procedentes de áreas sin alteraciones histológicas, los valores de IL-1 $\beta$ se encuentran significativamente elevados con respecto a los hallados en los controles sanos, aunque el incremento es menor al observado en el tejido alterado, un dato más que demuestra la presencia de valores elevados de mediadores inflamatorios más allá de las zonas histológicamente afectadas ${ }^{34}$.

La IL-18 tiene un importante papel en el mantenimiento de las respuestas Th1, tanto en enfermedades en humanos como en modelos experimentales en animales, con la potenciación de la expresión de IFN- $\gamma$ sinérgicamente con la $\mathrm{IL}-12^{50}$. Está presente tanto en la EC como en la CU, y se expresa en células epiteliales, en macrófagos activados de la lámina propia y en células dendríticas ${ }^{51}$ y, al igual que en el caso de otras citocinas inflamatorias, la principal fuente de IL-18 son los monocitos recién reclutados ${ }^{52}$. Esta citocina se secreta de forma inactiva y requiere que el ICE (enzima conversora de la IL-1 3 ) la escinda mediante proteólisis para generar la forma activa ${ }^{53}$. La IL-18 se encuentra presente en las zonas histológicamente afectadas y en las no afectadas tanto de la EC como de la CU, y los valores superiores se muestran en el caso de la $\mathrm{EC}^{51,52}$.

\section{Citocinas de la familia de la IL-12}

La IL-12 es un mediador de la respuesta inmunitaria frente a microorganismos intracelulares, así como un factor inductor clave de las respuestas de tipo Th1. La molécula de IL12 activa es un heterodímero formado por dos subunidades (p35 y p40). En condiciones normales, son varias poblaciones celulares las que sintetizan la subunidad p35, pero sólo las células presentadoras de antígeno profesionales y los linfocitos $T$ sintetizan el componente p40 y, por tanto, la citocina biológicamente activa. La IL-12 proporciona una importante conexión entre la inmunidad innata y la inmunidad adaptativa, ya que se produce durante las respuestas de inmunidad innata y estimula respuestas inmunitarias adaptativas al estimular la función de presentación de antígeno ${ }^{54,55}$. En la EC, las células mononucleares de la lámina propia, principalmente macrófagos, producen la IL-12, tanto en las zonas afectadas del intestino como en las no afectadas ${ }^{56}$; por el contrario, su presencia es muy reducida en la CU así como en el tejido sano $24,27,56$

La IL-12 y la IL-23 son dos citocinas relacionadas estructuralmente, ya que comparten la subunidad p40, aunque hay importantes diferencias en sus funciones. Mientras que la IL12 actúa en los linfocitos T vírgenes induciendo su polarización hacia el perfil Th1, la IL-23 actúa preferentemente en linfocitos T de memoria activados. Asimismo, la IL-23 produce también la estimulación de una subpoblación de linfocitos T productora de IL-17, una vía proinflamatoria independiente de IFN- $\gamma$ que se ha descubierto recientemente ${ }^{57,58}$. Se ha visto que los valores del ARNm de la IL-23 (p19) se encuentran elevados en la EC y se correlacionan con el grado de lesión; también están aumentados en la CU, aunque en menor medida que en el caso anterior ${ }^{59}$.

Las citocinas IL-27 e IL-12 muestran una gran proximidad estructural. La IL-27 está formada por la subunidad p28, homóloga de la IL-12 (p35) y por el Epstein-Barr virus-induced gene 3 (EBI3), una subunidad homóloga a IL-12 (p40). La IL-27 actúa en las células T vírgenes y las hace más sensibles a la polarización Th1 producida por IL-12. Estimula la acción proinflamatoria de las células CD4+, CD8+ y NK, al mismo tiempo que limita el grado de activación de esas células en modelos experimentales, como demuestra el hecho de que su ausencia produce un incremento significativo en la producción de otras citocinas ${ }^{57}$. Los valores de las dos subunidades de la IL-27 se encuentran elevados tanto en las áreas afectadas como en las no afectadas de la CU ${ }^{60}$ y los valores de EBI3 se correlacionan con el grado de afectación histológica en las zonas afectadas ${ }^{61}$. El papel de la IL-27 en la EC no está plenamente definido, ya que sólo un estudio detecta diferencias significativas en sus valores con respecto a los encontrados en los controles sanos ${ }^{59}$, mientras que otros dos trabajos no encuentran un incremento significativo en los valores de la IL-2760,61.

La subunidad p40, además de formar parte de las citocinas IL-12 e IL-23, también puede secretarse en forma dimérica. En modelos de colitis animal se ha comprobado que sus efectos varían según sea la concentración: cuando ésta es baja inhibe la producción de IFN- $\gamma$ y la proliferación de linfocitos, mientras que a concentraciones más elevadas tiene actividad proinflamatoria62. Otro estudio en un modelo de colitis animal ha llegado a conclusiones similares, ya que en ausencia de la subunidad p35 y, por tanto, incremento de 
la p40 dimérica, se induce una inflamación moderada asociada a una baja expresión de IL-18, mientras que la ausencia de p40, ya sea en ratones modificados genéticamente 0 bien mediante su bloqueo con anticuerpos específicos, se relacionaba con colitis grave y elevada expresión colónica de IL-1863.

\section{Mediadores de la inmunidad innata y adaptativa}

La IL-2 es un factor de crecimiento para los linfocitos T estimulados por antígeno y es la causa de la expansión clonal de las células $T$ tras el reconocimiento antigénico. También estimula la proliferación y la diferenciación de otras células inmunitarias como las células $\mathrm{NK}^{35}$. El ARNm de IL-2 aumenta en la EC únicamente en las zonas que muestran alteración histológica $27,64,65$ y, a diferencia de otras citocinas, los valores en las áreas no afectadas son similares a los observados en los controles sanos ${ }^{64}$. Prueba de la importancia de la IL-2 en la patogenia de la EC es la reducción observada en el número de células productoras de esta citocina tras el tratamiento con inmunodepresores y nutrición enteral en pacientes que han experimentado una mejoría clínica ${ }^{66}$. En el caso de la CU, los valores de la IL-2 no están aumentados en las biopsias de pacientes en actividad con respecto a los controles sanos ${ }^{64,65} y$, por tanto, esta citocina no estaría implicada en la patogenia de la CU.

La IL-15 es una citocina producida en respuesta a infecciones virales, componentes bacterianos como lipopolisacáridos (LPS) u otros factores que activan la inmunidad innata ${ }^{35}$. La función mejor documentada de la IL-15 es favorecer la proliferación y la activación de las células $N^{67}$, y podría considerarse que los efectos de la IL-15 en la respuesta innata son equivalentes a los de la IL-2 en la respuesta inmunitaria adaptativa. La IL-15 también puede actuar como factor de crecimiento y supervivencia de las células $T$, especialmente para las células T CD8+ de memoria de vida larga 68 , por lo que podría estar implicada en la supervivencia de los linfocitos específicos de memoria implicados en la inflamación intestinal. Esta citocina también desempeña un papel importante en la actividad del epitelio intestinal, ya que las células epiteliales pueden producir IL-15 y, al mismo tiempo, pueden proliferar frente a ella69. Tanto en la EC como en la CU se observa una expresión abundante de la IL-15 en las células mononucleares de la lámina propia, principalmente macrófagos activados, mientras que en el tejido procedente de controles sanos sólo se expresa de forma esporádica ${ }^{70,71}$. En cuanto a su expresión por las células epiteliales, los datos publicados son contradictorios, ya que un estudio mediante hibridación in situ muestra la expresión del ARNm de IL-15 por las células epiteliales, tanto en la EC como en la $\mathrm{CU}^{70}$, mientras que en otro los resultados mediante análisis inmunohistoquímico muestran la ausencia de células que expresan IL-15 en el epitelio ${ }^{71}$.

\section{Citocinas con función reguladora}

Los estudios sobre tolerancia oral y colitis inducidas experimentalmente en modelos animales indican que la inflamación gastrointestinal es el resultado de un balance entre citocinas proinflamatorias, principalmente IFN- $\gamma$, y otras de carácter opuesto, como TGF- $\beta^{16}$. También el balance entre los valores de IL-1 $\beta$ y su antagonista (IL-1Ra) refleja el grado de activación de los mecanismos inflamatorios en la mucosa intestinal, ya que se observa un gran aumento en la proporción de IL-1 $\beta$ en la Ell y, en menor medida, en otras inflamaciones menos graves, con respecto a los controles sanos ${ }^{47,72}$. El TGF- $\beta$ promueve la restitución del epitelio intestinal tras producirse un daño en la mucosa, y estimula el crecimiento y la diferenciación de células del mesénquima, como los fibroblastos $^{73}$. También tiene un papel importante en el recambio de la matriz extracelular al aumentar la síntesis de elastina, fibronectina y de distintos tipos de colágeno ${ }^{74}$. La expresión de TGF- $\beta$ aumenta en la mucosa inflamada, tanto de la EC como de la CU, mientras que en la mucosa intestinal de pacientes con Ell en remisión los valores de TGF- $\beta$ son similares a los encontrados en la mucosa normal. En el intestino normal se detecta ARNm de TGF- $\beta$ en la lámina propia y no en el epitelio; en la CU se expresa por células inflamatorias de la lámina propia próximas a la luz intestinal y en la EC la expresión se encuentra en toda la lámina propia, en especial, en zonas próximas al epitelio superficial. La presencia de valores más elevados de TGF- $\beta$ en la localización subepitelial indica que el TGF- $\beta$ tiene importancia en la reparación del epitelio que está justo encima, y estimula su restitución y el restablecimiento de la continuidad de la superficie epitelial tras la lesión ${ }^{75}$.

La IL-10 es una importante citocina inmunorreguladora que actúa en las células presentadoras de antígeno mediante la inhibición tanto de la síntesis de citocinas ${ }^{76}$ como de moléculas coestimuladoras ${ }^{77}$ y moléculas HLA de clase $11^{78}$. Asimismo, la IL-10 actúa directamente en la proliferación y la diferenciación de las células T. La activación de células T CD4+ humanas con células presentadoras de antígeno alogénicas en presencia de IL-10 produce anergia de larga duración específica de antígeno en las células T ${ }^{79}$. Además, en el hombre, la IL-10 induce la diferenciación de células T reguladoras de tipo I $(\operatorname{Tr} 1)^{80}$, población que se ha asociado con la patogenia de ciertas enfermedades autoinmunitarias ${ }^{81}$. En condiciones normales, las células epiteliales ${ }^{47,82}$ y las células mononucleares de la lámina propia mucosa ${ }^{46,47}$ expresan la IL-10. En las biopsias de pacientes con Ell se observa un grado de expresión de IL-10 en el epitelio similar al de los controles $\operatorname{sanos}^{47} \mathrm{y}$, al mismo tiempo, hay un incremento de la expresión de IL-10 en células mononucleares de la lámina propia ${ }^{27,47}$, principalmente en macrófagos recién reclutados ${ }^{46}$. El incremento en los valores de IL-10 es similar en la EC y en la CU, aunque podría no ser suficiente para poder llegar a controlar la inflamación ${ }^{47}$. Otra posible explicación a la falta de control de la inflamación sería que las células mononucleares de la lámina propia presentan una respuesta escasa a la acción antiinflamatoria de la IL-10, llegando incluso a promover la inflamación en presencia de la IL-12 ${ }^{83}$.

\section{Las citocinas como dianas terapéuticas en la EII}

Un mayor conocimiento de los mediadores implicados en la inflamación intestinal ha abierto la puerta a nuevas líneas de investigación basadas en la manipulación de la respuesta inmunitaria con fines terapéuticos (tabla 2), como son la neutralización de las citocinas proinflamatorias TNF- $\alpha^{84-86}$, IL-1287-89, IFN- $\gamma^{88}$ e IL-1890-92, el bloqueo del receptor de lá IL-693, así como la administración de la citocina reguladora IL-1094-96, o bien de la IL-11, que tiene efectos antiinflamatorios y promueve la regeneración del epitelio intestinal ${ }^{97-99}$. También se está ensayando el bloqueo de moléculas de adhesión, para así poder frenar la formación del infiltrado celular inflamatorio ${ }^{100,101}$

El infliximab es un anticuerpo monoclonal quimérico murino-humano frente al TNF- $\alpha$ que se desarrolló como agente terapéutico frente a las enfermedades mediadas por TNF$\alpha^{104}$. Los pacientes con EC presentan una respuesta rápida, aunque su duración es muy variable y puede llegar a producirse hipersensibilidad tras un uso prolongado ${ }^{4}$, así como producir sepsis ${ }^{105}$. Su mecanismo de acción, además de la 
LEÓN AJ ET AL. CITOCINAS EN LA PATOGENIA DE LA ENFERMEDAD INFLAMATORIA INTESTINAL

TABLA 2

\section{Nuevas aproximaciones terapéuticas en el tratamiento de la enfermedad inflamatoria intestinal}

\begin{tabular}{|c|c|c|}
\hline Aproximación terapéutica & Situación actual & Referencias \\
\hline Bloqueo de TNF- $\alpha$ & $\begin{array}{l}\text { Empleo de infliximab en la práctica clínica para el tratamiento de brotes de enfermedad } \\
\text { de Crohn }\end{array}$ & $84-86$ \\
\hline Administración de IL-10 & $\begin{array}{l}\text { Los ensayos clínicos demostraron que IL-10 es bien tolerado, aunque su utilidad } \\
\text { terapéutica es escasa }\end{array}$ & $94-96$ \\
\hline Administración de IL-11 & $\begin{array}{l}\text { En modelos de inflamación intestinal en animales, IL-11 reduce el daño de la mucosa. } \\
\text { Un ensayo clínico demuestra buena tolerancia y reducción del daño tisular }\end{array}$ & $97-99$ \\
\hline Bloqueo de IL-12 & $\begin{array}{l}\text { Estudios en animales y un ensayo clínico muestran la utilidad del bloqueo de la IL-12 } \\
\text { en el tratamiento de la enfermedad de Crohn }\end{array}$ & $87-89$ \\
\hline Bloqueo de IFN- $\gamma$ & $\begin{array}{l}\text { Estudios en modelos animales de colitis muestran reducción en la inflamación, } \\
\text { aunque menor que la obtenida con el bloqueo de IL-12 }\end{array}$ & 88 \\
\hline Bloqueo del receptor de IL-6 & $\begin{array}{l}\text { Un ensayo clínico muestra buena tolerancia al tratamiento, y mejora de los síntomas } \\
\text { durante la fase aguda de la inflamación }\end{array}$ & 93 \\
\hline Bloqueo de IL-18 & Estudios en modelos animales muestran atenuación de los síntomas & $90-92$ \\
\hline Bloqueo de IL-16 & Estudios en modelos animales muestran menor daño e inflamación & 103 \\
\hline $\begin{array}{l}\text { Inmunomodulación con huevos } \\
\text { de Trichuris suis }\end{array}$ & $\begin{array}{l}\text { Un estudio muestra la seguridad del tratamiento y la capacidad para modular la } \\
\text { respuesta inmunitaria, consiguiendo mejorar el estado clínico de los pacientes } \\
\text { de colitis ulcerosa }\end{array}$ & 102 \\
\hline
\end{tabular}

TNF- $\alpha$ : factor de necrosis tumoral alfa; IL: interleucina; IFN- $\gamma$ : interferón gamma; ICAM: molécula de adhesión intercelular.

unión al TNF- $\alpha$ soluble, parece estar relacionado con la inducción de apoptosis en las células que presentan TNF- $\alpha$ unido a su membrana ${ }^{104}$. Al contrario que en la EC, la utilidad del infliximab en pacientes con CU ha permanecido en entredicho durante largo tiempo, aunque estudios recientes postulan su utilidad en la CU refractaria ${ }^{106}$. Además del bloqueo de citocinas proinflamatorias, también se llevaron a cabo ensayos de administración de IL-10 humana94-96, aunque a pesar de que el tratamiento era bien tolerado, los beneficios resultaron ser escasos y se abandonó ${ }^{107}$.

En una nueva estrategia de inmunomodulación, se han utilizado huevos viables de Trichuris suis, un parásito que no desarrolla su ciclo vital en seres humanos, pero que produce una disminución en el grado de respuesta del sistema inmunitario intestinal en modelos de colitis experimental y, como también se ha visto en un ensayo clínico, inducen una mejoría en los síntomas clínicos en pacientes con CU ${ }^{106}$.

\section{Conclusiones}

La Ell es secundaria a una respuesta exagerada frente a la flora bacteriana en la mucosa intestinal, situación debida a factores ambientales, como el grado de higiene, y también a factores genéticos que incrementan el riesgo de desarrollar la enfermedad en las personas que portan determinados alelos de riesgo, especialmente en la EC y, en menor medida, en la CU. Los que se ocupan de regular el inicio, el mantenimiento y la remisión de la inflamación son, en último término, determinadas poblaciones de linfocitos $T$. En la Ell, los linfocitos T de la lámina propia se encuentran en un estado de activación superior al observado en condiciones normales y el patrón de citocinas producido es diferente en la EC, donde el perfil característico es de tipo Th1, con expresión de IFN- $\gamma$ y TNF- $\alpha$, mientras que en la CU el perfil es Th2, con citocinas como la IL-5. Una vez que se inicia el proceso inflamatorio, la principal fuente de citocinas son los macrófagos recién reclutados, que se encuentran en estado de activación. La pérdida en el equilibrio existente entre ci- tocinas proinflamatorias y antiinflamatorias, producidas por las distintas poblaciones celulares, es la causa de la inducción del daño tisular en la Ell.

El conocimiento de los mecanismos inmunológicos desencadenados en la Ell ha permitido identificar nuevas estrategias terapéuticas, mediante el bloqueo directo de citocinas proinflamatorias, o bien la administración de citocinas antiinflamatorias, aunque el grado de utilidad es diferente en cada situación. El grado de éxito obtenido varía desde el uso rutinario de infliximab hasta otros intentos con limitada utilidad terapéutica, como la administración de IL-10. Hay varias alternativas que se encuentran en distintas fases de experimentación, por lo que son necesarios más estudios para valorar la utilidad de estos tratamientos y garantizar su seguridad tras ser incorporadas a la práctica clínica.

\section{Agradecimientos}

Agradecemos al Prof. Blanco-Quirós la revisión crítica y los comentarios al manuscrito.

\section{REFERENCIAS BIBLIOGRÁFICAS}

1. Blumberg RS, Strober W. Prospects for research in inflammatory bowel disease. JAMA. 2001;285:643-7.

2. Ekbom A, Helmick C, Zack M, Adami HO. Ulcerative colitis and colorectal cancer. A population-based study. N Engl J Med. 1990;323:1228-33.

3. Shanahan F. Crohn's disease. Lancet. 2002;359:62-9

4. Podolsky DK. Inflammatory bowel disease. N Engl J Med. 2002; 347:417-29.

5. Sutherland L, Singleton J, Sessions J, Hanauer S, Krawitt E, Rankin G, et al. Double blind, placebo controlled trial of metronidazole in Crohn's disease. Gut. 1991;32:1071-5.

6. Rembacken BJ, Snelling AM, Hawkey PM, Chalmers DM, Axon AT. Non-pathogenic Escherichia coli versus mesalazine for the treatment of ulcerative colitis: a randomised trial. Lancet. 1999;354:635-9.

7. Shanahan F. Probiotics and inflammatory bowel disease: is there a scientific rationale? Inflamm Bowel Dis. 2000;6:107-15.

8. Elliott DE, Urban JF Jr, Argo CK, Weinstock JV. Does the failure to acquire helminthic parasites predispose to Crohn's disease? FASEB J. 2000; $14: 1848-55$

9. Theis MK, Boyko EJ. Patient perceptions of causes of inflammatory bowel disease. Am J Gastroenterol. 1994;89:1920. 
LEÓN AJ ET AL. CITOCINAS EN LA PATOGENIA DE LA ENFERMEDAD INFLAMATORIA INTESTINAL

10. Tysk C, Lindberg E, Jarnerot G, Floderus-Myrhed B. Ulcerative colitis and Crohn's disease in an unselected population of monozygotic and dizygotic twins. A study of heritability and the influence of smoking. Gut. 1988;29:990-6.

11. Orholm M, Binder V, Sorensen TI, Rasmussen LP, Kyvik KO. Concordance of inflammatory bowel disease among Danish twins. Results of a nationwide study. Scand J Gastroenterol. 2000;35:1075-81.

12. Roussomoustakaki M, Satsangi J, Welsh K, Louis E, Fanning G, Targan $\mathrm{S}$, et al. Genetic markers may predict disease behavior in patients with ulcerative colitis. Gastroenterology. 1997;112:1845-53.

13. Lesage S, Zouali H, Cezard JP, Colombel JF, Belaiche J, Almer S, et al. CARD15/NOD2 mutational analysis and genotype-phenotype correlation in 612 patients with inflammatory bowel disease. Am J Hum Genet. 2002;70:845-57.

14. Futami S, Aoyama N, Honsako Y, Tamura T, Morimoto S, Nakashima T, et al. HLA-DRB1*1502 allele, subtype of DR15, is associated with susceptibility to ulcerative colitis and its progression. Dig Dis Sci. 1995;40:814-8.

15. MacDonald TT, Monteleone G. IL-12 and Th1 immune responses in human Peyer's patches. Trends Immunol. 2001;22:244-7.

16. Strober W, Kelsall B, Fuss I, Marth T, Ludviksson B, Ehrhardt R, et al. Reciprocal IFN-gamma and TGF-beta responses regulate the occurrence of mucosal inflammation. Immunol Today. 1997; 18:61-4.

17. Boirivant M, Marini M, Di FG, Pronio AM, Montesani C, Tersigni R, et al. Lamina propria T cells in Crohn's disease and other gastrointestinal inflammation show defective CD2 pathway-induced apoptosis. Gastroinflammation show defective CD2

18. Atreya R, Mudter J, Finotto S, Mullberg J, Jostock T, Wirtz S, et al. Blockade of interleukin 6 trans signaling suppresses T-cell resistance against apoptosis in chronic intestinal inflammation: evidence in Crohn's disease and experimental colitis in vivo. Nat Med. 2000;6:583-8.

19. Mariani P, Bachetoni A, D'Alessandro M, Lomanto D, Mazzocchi P, Speranza V. Effector Th-1 cells with cytotoxic function in the intestinal lamina propria of patients with Crohn's disease. Dig Dis Sci. 2000:45:2029-35.

20. Powrie F. T cells in inflammatory bowel disease: protective and pathogenic roles. Immunity. 1995;3:171-4.

21. Makita S, Kanai T, Oshima S, Uraushihara K, Totsuka T, Sawada T, et al. CD4+CD25bright T cells in human intestinal lamina propria as regulatory cells. J Immunol. 2004:173:3119-30.

22. Fuss IJ, Neurath M, Boirivant M, Klein JS, De la Motte C, Strong SA, et al. Disparate CD4+ lamina propria (LP) lymphokine secretion profiles in inflammatory bowel disease. Crohn's disease LP cells manifest increased secretion of IFN-gamma, whereas ulcerative colitis LP cells manifest increased secretion of IL-5. J Immunol. 1996;157:1261-70.

23. Agnholt J, Kaltoft K. In situ activated intestinal T cells expanded in vitro-without addition of antigen-produce IFN-gamma and IL-10 and preserve their function during growth. Exp Clin Immunogenet. 2001;18:213-25

24. Parronchi P, Romagnani $P$, Annunziato F, Sampognaro S, Becchio A, Giannarini L, et al. Type 1 T-helper cell predominance and interleukin12 expression in the gut of patients with Crohn's disease. Am J Pathol. 1997:150:823-32.

25. Van Damme N, De Vos M, Demetter P, Baeten D, Mielants H, Verbruggen $\mathrm{G}$, et al. The proportion of Th1 cells, which prevail in gut mucosa, is decreased in inflammatory bowel syndrome. Clin Exp Immunol. 2001;125:383-90.

26. Autschbach F, Giese T, Gassler N, Sido B, Heuschen G, Heuschen U, et al. Cytokine/chemokine messenger-RNA expression profiles in ulcerative colitis and Crohn's disease. Virchows Arch. 2002;441:500-13.

27. Kakazu T, Hara J, Matsumoto T, Nakamura S, Oshitani N, Arakawa T, et al. Type 1 T-helper cell predominance in granulomas of Crohn's disease. Am J Gastroenterol. 1999;94:2149-55.

28. Reimund JM, Wittersheim C, Dumont S, Muller CD, Kenney JS, Baumann R, et al. Increased production of tumour necrosis factor-alpha interleukin-1 beta, and interleukin- 6 by morphologically normal intestinal biopsies from patients with Crohn's disease. Gut. 1996:39:684-9.

29. Goodman MJ, Skinner JM, Truelove SC. Abnormalities in the apparently normal bowel mucosa in Crohn's disease. Lancet. 1976;1:275-8.

30. Dunne WT, Cooke WT, Allan RN. Enzymatic and morphometric evidence for Crohn's disease as a diffuse lesion of the gastrointestinal tract. Gut. 1977;18:290-4.

31. Ahrenstedt O, Knutson L, Nilsson B, Nilsson-Ekdahl K, Odlind B, Hallgren $R$. Enhanced local production of complement components in the small intestines of patients with Crohn's disease. N Engl J Med. 1990;322:1345-9.

32. Olaison G, Sjodahl R, Tagesson C. Increased phospholipase A2 activity of Ileal mucosa in Crohn's disease. Digestion. 1988;41:136-41.

33. Nagel E, Bartels M, Pichlmayr R. Scanning electron-microscopic lesions in Crohn's disease: relevance for the interpretation of postoperative recurrence. Gastroenterology. 1995;108:376-82.

34. Dionne S, Hiscott J, D'Agata I, Duhaime A, Seidman EG. Quantitative PCR analysis of TNF-alpha and IL-1 beta mRNA levels in pediatric IBD mucosal biopsies. Dig Dis Sci. 1997;42:1557-66.

35. Abbas AK LAPJ. Inmunología celular y molecular. Cuarta edición ed. Madrid: Interamericana-McGraw-Hill; 2002.

36. Boehm U, Klamp T, Groot M, Howard JC. Cellular responses to interferon-gamma. Annu Rev Immunol. 1997;15:749-95.
37. MacDonald TT. Effector and regulatory lymphoid cells and cytokines in mucosal sites. Curr Top Microbiol Immunol. 1999;236:113-35.

38. Leon AJ, Garrote JA, Arranz E. Citocinas en la patogenia de la enfermedad celíaca. Med Clin (Barc). 2005;125:508-16.

39. Neurath MF, Finotto S, Glimcher LH. The role of Th1/Th2 polarization in mucosal immunity. Nat Med. 2002:8:567-73.

40. Schreiber S, Rosenstiel P, Hampe J, Nikolaus S, Groessner B, Schottelius $A$, et al. Activation of signal transducer and activator of transcription (STAT) 1 in human chronic inflammatory bowel disease. Gut. 2002;51:379-85.

41. Gibson PR. Increased gut permeability in Crohn's disease: is TNF the link? Gut. 2004;53:1724-5.

42. Guy-Grand D, DiSanto JP, Henchoz P, Malassis-Seris M, Vassalli P. Small bowel enteropathy: role of intraepithelial lymphocytes and of cytokines (IL-12, IFN-gamma, TNF) in the induction of epithelial cell death and renewal. Eur J Immunol. 1998;28:730-44.

43. Kaiser GC, Polk DB. Tumor necrosis factor alpha regulates proliferation in a mouse intestinal cell line. Gastroenterology. 1997;112:1231-40.

44. Breese EJ, Michie CA, Nicholls SW, Murch SH, Williams CB, Domizio $\mathrm{P}$, et al. Tumor necrosis factor alpha-producing cells in the intestinal mucosa of children with inflammatory bowel disease. Gastroenterology. 1994;106:1455-66.

45. Akazawa A, Sakaida I, Higaki S, Kubo Y, Uchida K, Okita K. Increased expression of tumor necrosis factor-alpha messenger RNA in the intestinal mucosa of inflammatory bowel disease, particularly in patients with disease in the inactive phase. J Gastroenterol. 2002;37:345-53.

46. Rugtveit J, Nilsen EM, Bakka A, Carlsen H, Brandtzaeg P, Scott H. Cytokine profiles differ in newly recruited and resident subsets of mucosal macrophages from inflammatory bowel disease. Gastroenterology. 1997; 112:1493-505.

47. Autschbach F, Braunstein J, Helmke B, Zuna I, Schurmann G, Niemir $\mathrm{ZI}$, et al. In situ expression of interleukin-10 in noninflamed human gut and in inflammatory bowel disease. Am J Pathol. 1998;153:121-30.

48. McAlindon ME, Hawkey CJ, Mahida YR. Expression of interleukin 1 beta and interleukin 1 beta converting enzyme by intestinal macrophages in health and inflammatory bowel disease. Gut. 1998;42:214-9.

49. Reinecker HC, Steffen M, Witthoeft T, Pflueger I, Schreiber S, MacDermott RP, et al. Enhanced secretion of tumour necrosis factor-alpha, IL6 , and IL-1 beta by isolated lamina propria mononuclear cells from patients with ulcerative colitis and Crohn's disease. Clin Exp Immunol. 1993;94:174-81.

50. Robinson D, Shibuya K, Mui A, Zonin F, Murphy E, Sana T, et al. IGIF does not drive Th1 development but synergizes with IL-12 for interferon-gamma production and activates IRAK and NFkappaB. Immunity. 1997;7:571-81.

51. Pizarro TT, Michie MH, Bentz M, Woraratanadharm J, Smith MF Jr, Foley E, et al. IL-18, a novel immunoregulatory cytokine, is up-regulated in Crohn's disease: expression and localization in intestinal mucosal cells. J Immunol. 1999;162:6829-35.

52. Monteleone G, Trapasso F, Parrello T, Biancone L, Stella A, Iuliano R, et al. Bioactive IL-18 expression is up-regulated in Crohn's disease. J Immunol. 1999;163:143-7.

53. Ghayur T, Banerjee S, Hugunin M, Butler D, Herzog L, Carter A, et al. Caspase-1 processes IFN-gamma-inducing factor and regulates LPSinduced IFN-gamma production. Nature. 1997;386:619-23.

54. Watford WT, Moriguchi M, Morinobu A, O'Shea JJ. The biology of IL12: coordinating innate and adaptive immune responses. Cytokine Growth Factor Rev. 2003;14:361-8.

55. Trinchieri G. Interleukin-12 and the regulation of innate resistance and adaptive immunity. Nat Rev Immunol. 2003;3:133-46.

56. Monteleone G, Biancone L, Marasco R, Morrone G, Marasco O, Luzza $F$, et al. Interleukin 12 is expressed and actively released by Crohn's disease intestinal lamina propria mononuclear cells. Gastroenterology. 1997; 112:1169-78.

57. Hunter CA. New IL-12-family members: IL-23 and IL-27, cytokines with divergent functions. Nat Rev Immunol. 2005;5:521-31.

58. Langrish CL, McKenzie BS, Wilson NJ, De Waal MR, Kastelein RA, Cua DJ. IL-12 and IL-23: master regulators of innate and adaptive immunity. Immunol Rev. 2004;202:96-105.

59. Schmidt C, Giese T, Ludwig B, Mueller-Molaian I, Marth T, Zeuzem S, et al. Expression of interleukin-12-related cytokine transcripts in inflammatory bowel disease: elevated interleukin-23p19 and interleukin27 p28 in Crohn's disease but not in ulcerative colitis. Inflamm Bowel Dis. 2005; 11:16-23

60. Omata F, Birkenbach M, Matsuzaki S, Christ AD, Blumberg RS. The expression of IL-12 p40 and its homologue, Epstein-Barr virus-induced gene 3 , in inflammatory bowel disease. Inflamm Bowel Dis. 2001;7:215-20.

61. Christ AD, Stevens AC, Koeppen H, Walsh S, Omata F, Devergne O, et al. An interleukin 12-related cytokine is up-regulated in ulcerative colitis but not in Crohn's disease. Gastroenterology. 1998;115:307-13.

62. Stallmach A, Marth T, Weiss B, Wittig BM, Hombach A, Schmidt C, et al. An interleukin 12 p40-lgG2b fusion protein abrogates T cell mediated inflammation: anti-inflammatory activity in Crohn's disease and experimental colitis in vivo. Gut. 2004;53:339-45.

63. Camoglio L, Juffermans NP, Peppelenbosch M, Te Velde AA, Ten Kate FJ, Van Deventer SJ, et al. Contrasting roles of IL-12p40 and IL-12p35 in the development of hapten-induced colitis. Eur J Immunol. 2002;32:261-9. 
LEÓN AJ ET AL. CITOCINAS EN LA PATOGENIA DE LA ENFERMEDAD INFLAMATORIA INTESTINAL

64. Mullin GE, Lazenby AJ, Harris ML, Bayless TM, James SP. Increased interleukin-2 messenger RNA in the intestinal mucosal lesions of Crohn's disease but not ulcerative colitis. Gastroenterology. 1992;102:1620-7.

65. Niessner M, Volk BA. Altered Th1/Th2 cytokine profiles in the intestinal mucosa of patients with inflammatory bowel disease as assessed by quantitative reversed transcribed polymerase chain reaction (RT-PCR). Clin Exp Immunol. 1995;101:428-35.

66. Breese EJ, Michie CA, Nicholls SW, Williams CB, Domizio P, WalkerSmith JA, et al. The effect of treatment on lymphokine-secreting cells in the intestinal mucosa of children with Crohn's disease. Aliment Pharmacol Ther. 1995:9:547-52.

67. Mrozek E, Anderson P, Caligiuri MA. Role of interleukin-15 in the development of human CD56+ natural killer cells from CD34+ hematopoietic progenitor cells. Blood. 1996;87:2632-40.

68. Kanegane $\mathrm{H}$, Tosato G. Activation of naive and memory $\mathrm{T}$ cells by interleukin-15. Blood. 1996;88:230-5.

69. Reinecker HC, MacDermott RP, Mirau S, Dignass A, Podolsky DK. Intestinal epithelial cells both express and respond to interleukin 15. Gastroenterology. 1996;111:1706-13

70. Sakai T, Kusugami K, Nishimura H, Ando T, Yamaguchi T, Ohsuga M, et al. Interleukin 15 activity in the rectal mucosa of inflammatory bowel disease. Gastroenterology. 1998;114:1237-43.

71. Liu Z, Geboes K, Colpaert S, D'Haens GR, Rutgeerts P, Ceuppens JL. IL15 is highly expressed in inflammatory bowel disease and regulates local T cell-dependent cytokine production. J Immunol. 2000;164:3608-15.

72. Dionne S, D'Agata ID, Hiscott J, Vanounou T, Seidman EG. Colonic explant production of IL-1and its receptor antagonist is imbalanced in inflammatory bowel disease (IBD). Clin Exp Immunol. 1998;112:435-42.

73. Pierce GF, Mustoe TA, Lingelbach J, Masakowski VR, Griffin GL, Senio $\mathrm{RM}$, et al. Platelet-derived growth factor and transforming growth factor-beta enhance tissue repair activities by unique mechanisms. J Cell Biol. 1989; 109:429-40.

74. Barnard JA CRJ. Transforming growth factor [beta]. En: Walsh JH, Dockray GJ, editors. Gut peptides: Biochemistry and physiology. New York: Raven Press; 1993. p. 615-31.

75. Babyatsky MW, Rossiter G, Podolsky DK. Expression of transforming growth factors alpha and beta in colonic mucosa in inflammatory bowe disease. Gastroenterology. 1996;110:975-84.

76. Fiorentino DF, Zlotnik A, Vieira P, Mosmann TR, Howard M, Moore $\mathrm{KW}$, et al. IL-10 acts on the antigen-presenting cell to inhibit cytokine production by Th1 cells. J Immunol. 1991;146:3444-51.

77. Buelens C, Willems F, Delvaux A, Pierard G, Delville JP, Velu T, et al Interleukin-10 differentially regulates B7-1 (CD80) and B7-2 (CD86) expression on human peripheral blood dendritic cells. Eur J Immunol. 1995;25:2668-72.

78. De Waal MR, Haanen J, Spits H, Roncarolo MG, Te VA, Figdor C, et al. Interleukin 10 (IL-10) and viral IL-10 strongly reduce antigen-specific human $T$ cell proliferation by diminishing the antigen-presenting capacity of monocytes via downregulation of class II major histocompatibility complex expression. J Exp Med. 1991;174:915-24.

79. Groux H, Bigler M, De Vries JE, Roncarolo MG. Interleukin-10 induces a long-term antigen-specific anergic state in human CD4+ T cells. Exp Med. 1996:184:19-29.

80. Jonuleit $H$, Schmitt E. The regulatory $T$ cell family: distinct subsets and their interrelations. J Immunol 2003;171:6323-7.

81. Yudoh K, Matsuno H, Nakazawa F, Yonezawa T, Kimura T. Reduced expression of the regulatory CD4+ T cell subset is related to Th1/Th2 balance and disease severity in rheumatoid arthritis. Arthritis Rheum. 2000;43:617-27.

82. Napolitano LM, Buzdon MM, Shi HJ, Bass BL. Intestinal epithelial cell regulation of macrophage and lymphocyte interleukin 10 expression. Arch Surg. 1997;132:1271-6.

83. Colpaert S, Vanstraelen K, Liu Z, Penninckx F, Geboes K, Rutgeerts P, et al. Decreased lamina propria effector cell responsiveness to interleukin-10 in ileal Crohn's disease. Clin Immunol. 2002;102:68-76.

84. Rutgeerts $P$, Van AG, Vermeire S. Optimizing anti-TNF treatment in inflammatory bowel disease. Gastroenterology. 2004;126:1593-610.

85. Cadahia V, Garcia-Carbonero A, Vivas S, Fuentes D, Nino P, Rebollo P, et al. Infliximab improves quality of life in the short-term in patients with fistulizing Crohn's disease in clinical practice. Rev Esp Enferm Dig. 2004;96:369-74.
86. Baert FJ, D'Haens GR, Peeters M, Hiele MI, Schaible TF, Shealy D, et al. Tumor necrosis factor alpha antibody (infliximab) therapy profoundly down-regulates the inflammation in Crohn's ileocolitis. Gastroenterology. 1999;116:22-8.

87. Neurath MF, Fuss I, Kelsall BL, Stuber E, Strober W. Antibodies to interleukin 12 abrogate established experimental colitis in mice. J Exp Med. 1995;182:1281-90.

88. Fuss IJ, Marth T, Neurath MF, Pearlstein GR, Jain A, Strober W. Antiinterleukin 12 treatment regulates apoptosis of Th1 T cells in experimental colitis in mice. Gastroenterology. 1999;117:1078-88.

89. Mannon PJ, Fuss IJ, Mayer L, Elson CO, Sandborn WJ, Present D, et al. Anti-interleukin-12 antibody for active Crohn's disease. N Engl J Med. 2004;351:2069-79.

90. Sivakumar PV, Westrich GM, Kanaly S, Garka K, Born TL, Derry JM, et al. Interleukin 18 is a primary mediator of the inflammation associated with dextran sulphate sodium induced colitis: blocking interleukin 18 attenuates intestinal damage. Gut. 2002;50:812-20.

91. Kanai T, Watanabe M, Okazawa A, Sato T, Yamazaki M, Okamoto S, et al. Macrophage-derived IL-18-mediated intestinal inflammation in the murine model of Crohn's disease. Gastroenterology. 2001;121:875-88.

92. Ten HT Corbaz A, Amitai H, Aloni S, Belzer I, Graber P et al. Blockade of endogenous IL-18 ameliorates TNBS-induced colitis by decreasing local TNF-alpha production in mice. Gastroenterology. 2001;121:1372-9.

93. Ito $\mathrm{H}$, Takazoe M, Fukuda $\mathrm{Y}$, Hibi T, Kusugami K, Andoh A, et al. A pilot randomized trial of a human anti-interleukin-6 receptor monoclonal antibody in active Crohn's disease. Gastroenterology. 2004;126:989-96.

94. Schreiber S, Fedorak RN, Nielsen OH, Wild G, Williams CN, Nikolaus $\mathrm{S}$, et al. Safety and efficacy of recombinant human interleukin 10 in chronic active Crohn's disease. Crohn's Disease IL-10 Cooperative Study Group. Gastroenterology. 2000;119:1461-72.

95. Fedorak RN, Gangl A, Elson CO, Rutgeerts P, Schreiber S, Wild G, et al. Recombinant human interleukin 10 in the treatment of patients with mild to moderately active Crohn's disease. The Interleukin 10 Inflammatory Bowel Disease Cooperative Study Group. Gastroenterology. 2000;119:1473-82.

96. Colombel JF, Rutgeerts $\mathrm{P}$, Malchow $\mathrm{H}$, Jacyna M, Nielsen $\mathrm{OH}$, RaskMadsen J, et al. Interleukin 10 (Tenovil) in the prevention of postoperative recurrence of Crohn's disease. Gut. 2001;49:42-6.

97. Sands BE, Bank S, Sninsky CA, Robinson M, Katz S, Singleton JW, et al. Preliminary evaluation of safety and activity of recombinant human interleukin 11 in patients with active Crohn's disease. Gastroenterology. 1999; 117:58-64.

98. Sonis S, Muska A, O'Brien J, Van VA, Langer-Safer P, Keith J. Alteration in the frequency, severity and duration of chemotherapy-induced mucositis in hamsters by interleukin-11. Eur J Cancer B Oral Oncol. 1995;31B:261-6.

99. Sonis ST, Van Vugt AG, McDonald J, Dotoli E, Schwertschlag U, Szklut P, et al. Mitigating effects of interleukin 11 on consecutive courses of 5-fluorouracil-induced ulcerative mucositis in hamsters. Cytokine. 1997;9:605-12.

100. Yacyshyn BR, Bowen-Yacyshyn MB, Jewell L, Tami JA, Bennett CF, Kisner $\mathrm{DL}$, et al. A placebo-controlled trial of ICAM-1 antisense oligonucleotide in the treatment of Crohn's disease. Gastroenterology. 1998;114:1133-42.

101. Sans M, Salas A, Soriano A, Prats N, Gironella M, Pizcueta P, et al. Differential role of selectins in experimental colitis. Gastroenterology. 2001;120:1162-72.

102. Summers RW, Elliott DE, Urban JF Jr., Thompson RA, Weinstock JV Trichuris suis therapy for active ulcerative colitis: a randomized controIled trial. Gastroenterology. 2005;128:825-32.

103. Keates AC, Castagliuolo I, Cruickshank WW, Qiu B, Arseneau KO, Brazer W, et al. Interleukin 16 is up-regulated in Crohn's disease and participates in TNBS colitis in mice. Gastroenterology. 2000;119:972-82.

104. Knight DM, Trinh H, Le J, Siegel S, Shealy D, McDonough M, et al. Construction and initial characterization of a mouse-human chimeric anti-TNF antibody. Mol Immunol. 1993;30:1443-53.

105. De la Fuente PB, Marco PA, Riera MA, Boadas MJ. Sepsis por Listeria monocytogenes asociada al uso de infliximab. Med Clin (Barc). 2005:124:398.

106. D'Haens G. Infliximab for ulcerative colitis: finally some answers. Gastroenterology. 2005;128:2161-4.

107. Sandborn WJ, Targan SR. Biologic therapy of inflammatory bowel disease. Gastroenterology. 2002;122:1592-608. 